\title{
Optical response of monolayer films of a metal-free sulfonamido-porphyrin
}

\author{
T. Richardson ${ }^{a} *$, V.C. Smith ${ }^{\mathrm{a}}$, R.A.W. Johnstone ${ }^{\mathrm{b}}$, \\ A.J.F.N. Sobral ${ }^{\mathrm{c}}$, A.M.d'A. Rocha-Gonsalves ${ }^{\mathrm{c}}$ \\ ${ }^{a}$ Department of Physics, University of Sheffield, Hounsfield Road, Sheffield S3 7RH, UK \\ ${ }^{\mathrm{b}}$ Department of Chemistry, University of Liverpool, Liverpool L69 3BX, UK \\ ${ }^{\mathrm{c}}$ Departamento de Quimica, Universidade de Coimbra, Coimbra, Portugal
}

\begin{abstract}
The influence of gaseous chlorine on monolayers of metal-free meso-tetra(4-amino sulfonyl)phenylporphyrin is discussed. These films exhibit characteristic intense optical absorption bands in the region 400-700 $\mathrm{nm}$. Upon exposure to chlorine vapour in the concentration range 0.1-10 ppm, the Soret and Q-bands decrease in intensity and new bands at $\sim 455 \mathrm{~nm}$ and $\sim 660 \mathrm{~nm}$ appear. The rate of response has been measured as a function of deposition surface pressure and gas concentration. Repeated dosing experiments have shown that reversible and reproducible sensing action occurs provided that a threshold concentration is not exceeded. The recovery of the original optical spectrum can be accelerated with moderate heat treatment $\left(60^{\circ} \mathrm{C}\right.$ for $\left.10 \mathrm{~min}\right)$. Ageing experiments have shown that the basic response of the monolayer is not effected over a time period of at least 5 months. (c) 1998 Elsevier Science S.A. All rights reserved
\end{abstract}

Keywords: Chlorine; Porphyrin; Langmuir-Blodgett

\section{Introduction}

Electronic interactions occurring between adjacent extended $\pi$-electron systems are important in various systems such as photosynthetic light harvesting entities and molecular (semi)conductors. Examples of such materials are porphyrins and phthalocyanines which have been shown to display environment-sensitive physical properties [1]. In particular, several metal phthalocyanines have attracted interest as a result of the sensitivity of their resistivity to low concentrations of toxic gases such as nitrogen dioxide [2]. Relatively little attention, however, has been paid to the detection of chlorine gas using organic sensing agents. Utilisation of the optical absorption spectrum as a sensing parameter is attractive, since sample preparation is extremely straightforward and does not involve the fabrication of intricate interdigitated electrode patterns usually necessary in conductometric sensors [3]. This simple approach demands lower electrical power than its resistive counterpart which requires the continual passage of electrical current through the sample itself. This can result in

\footnotetext{
* Corresponding author. Tel.: +44 114 2224280; fax: +44 114 2728079; e-mail: t.richardson@sheffield.ac.uk
}

gradual degradation of the sensing film. In this paper, a substituted porphyrin compound is used to detect chlorine gas in the concentration range $0.1-10 \mathrm{ppm}$.

\section{Experimental}

\subsection{Langmuir and Langmuir-Blodgett (LB) film preparation}

The porphyrin studied is a metal-free sulfonamidophenyl porphyrin (t-SAP-P) whose chemical structure is shown in Fig. 1a. Its synthesis has been described previously [4]. t-SAP-P was spread on the subphase surface (pure water, $\mathrm{pH} 6.0,20^{\circ} \mathrm{C}$ ) from a solution of concentration $0.08 \mathrm{mg} \mathrm{ml}^{-1}$ in chloroform. The surface pressure-area isotherm was recorded using a constant-perimeter Langmuir trough using a compression rate of $\sim 7 \mathrm{~cm}^{2} \mathrm{~s}^{-1}$ (maximum trough area $=900 \mathrm{~cm}^{2}$ ).

\subsection{Exposure to chlorine gas}

A purpose-built gas chamber and chlorine gas generator were used in conjunction with a multi-channel photodiode array spectrophotometer (Otsuka Electronics, Japan) in 
order to study the optical response of the LB monolayers upon exposure to low concentrations of chlorine. Fig. 2 shows the experimental arrangement. Electrolysis of $2 \mathrm{M}$ aqueous $\mathrm{HCl}$ results in the generation of chlorine gas which is blown into the sample chamber by a motor-driven fan. Three commercially available chlorine sensors (CiTiCell) surround the LB film in the sample chamber to ensure an representative chlorine concentration is measured. A feedback connection between the sensor outputs and the power supply to the electrolysis cell enables the preset chlorine concentration to be maintained throughout each experiment. The fast photodiode array means that several spectra (over the wavelength range typically $350-800 \mathrm{~nm}$ ) were averaged in under $0.5 \mathrm{~s}$ corresponding to each measurement. The spectral changes were monitored typically over a 30-min time period during exposure, even though saturation often occurred much sooner. The recovery in air of the optical spectrum after exposure was also monitored at ambient temperature $\left(\sim 20^{\circ} \mathrm{C}\right)$ and at $60^{\circ} \mathrm{C}$. Repeated dosing experiments were performed by initially exposing the monolayer to chlorine for $10 \mathrm{~min}$ followed by a long time period for recovery. A second exposure to chlorine was exacted $20 \mathrm{~h}$ after the initial exposure and so on. Long-term ageing experiments involved storing an unexposed monolayer film in the dark under ambient conditions for a 5-month time period and then comparing its response and recovery characteristics to a freshly prepared monolayer.

\section{Results and discussion}

\subsection{Surface pressure-area isotherm}

The surface pressure-area isotherm, given in Fig. 1b, shows that three distinct phases are observed. At high area per molecule, the surface pressure rises gradually to $11 \mathrm{mN}$ $\mathrm{m}^{-1}$ (the low-pressure region) at which point there occurs a phase transition to a plateau region. A second phase transition occurs at $12 \mathrm{mN} \mathrm{m}^{-1}$ to a less compressible phase (the high-pressure region). Deposition occurred on withdrawal of the substrate (glass plate) through the air/water interface throughout the low- and high-pressure regions. The deposition speed was $4 \mathrm{~mm} \mathrm{~min}^{-1}$. Earlier orientation studies using polarised optical spectroscopy [5] indicated that at low pressure the porphyrin molecules lie flat and plate-like at the water surface, and at high pressure the molecules are forced into a tilted arrangement with the central tetraphenyl porphyrin core at approximately $30^{\circ}$ to the plane of the surface.

\subsection{Dependence of the gas response on the deposition surface pressure}

Fig. 3a shows the gas response for a monolayer deposited at $8 \mathrm{mN} \mathrm{m}^{-1}$ (low pressure region) during exposure to $5 \mathrm{ppm}$ chlorine. A selection of spectra are shown over the 30-min exposure. Clearly the Soret band at $420 \mathrm{~nm}$ falls with the dramatic emergence of a new band at $455 \mathrm{~nm}$ which reaches nearly half its final intensity after only $30 \mathrm{~s}$. The band at 455 nm shifts slightly to higher wavelength during the exposure; this minor effect is presently not understood. Another band at $\sim 660 \mathrm{~nm}$ also grows in intensity during the exposure. A logarithmic plot of the normalised Soret band absorbance (i.e. $\ln I_{\mathrm{N}}=\log _{\mathrm{e}}$ (absorbance at time $t /$ initial absorbance at $t=0$ ) versus time shows that a well-defined initial rate constant characterises the gas response over the first $60 \mathrm{~s}$ but then the kinetics become more complex. Clearly the kinetic process needs to be modelled in detail, but for the purpose of comparison, the constant $k$ is useful and within the scope of this short paper. A similar study for a monolayer deposited in the high pressure region $\left(16 \mathrm{mN} \mathrm{m}^{-1}\right)$ shows the same features although they are much less pronounced (Fig. 3b). The deposition pressure at which the highest initial rate constant occurs and for which the Soret band intensity falls to $50 \%$ of its original value in the shortest time period of only $60 \mathrm{~s}$ is $8 \mathrm{nM} \mathrm{m}^{-1}$. The corresponding half-intensity time periods for monolayer samples deposited at surface pressures at or above $16 \mathrm{mN} \mathrm{m}^{-1}$ are at least an order of magnitude longer. This suggests that the porphyrin molecules are most susceptible to adsorption when deposited at a

(a)
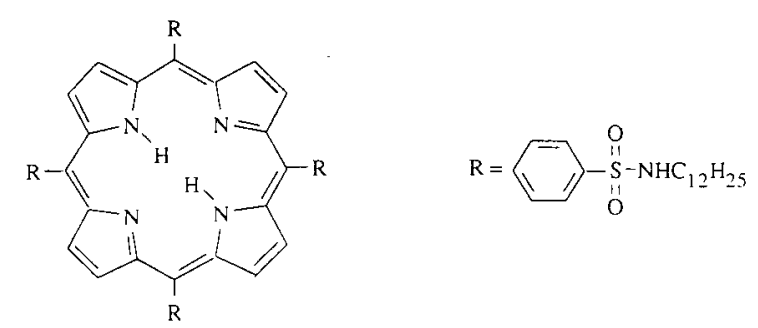

(b)

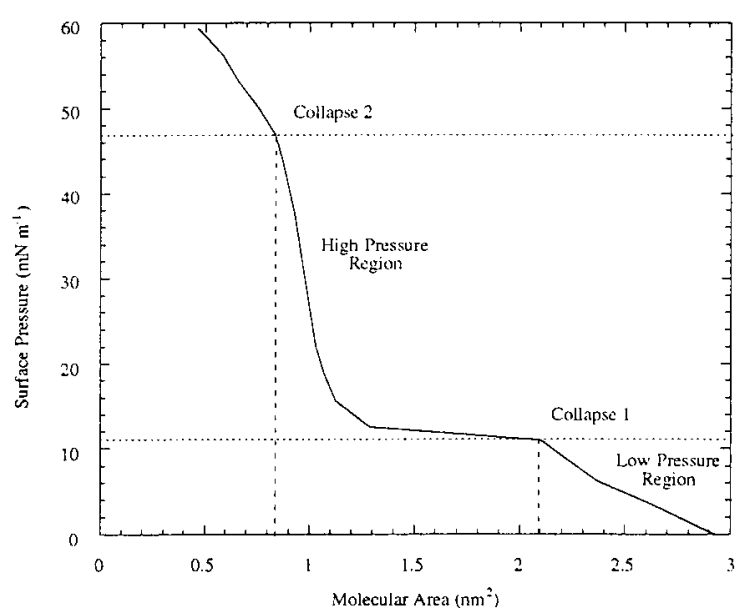

Fig. 1. (a) Chemical structure of metal-free tetra-sulfonylamidophenyl porphyrin (t-SAP-P); (b) Surface pressure-molecular area isotherm for tSAP-P. 

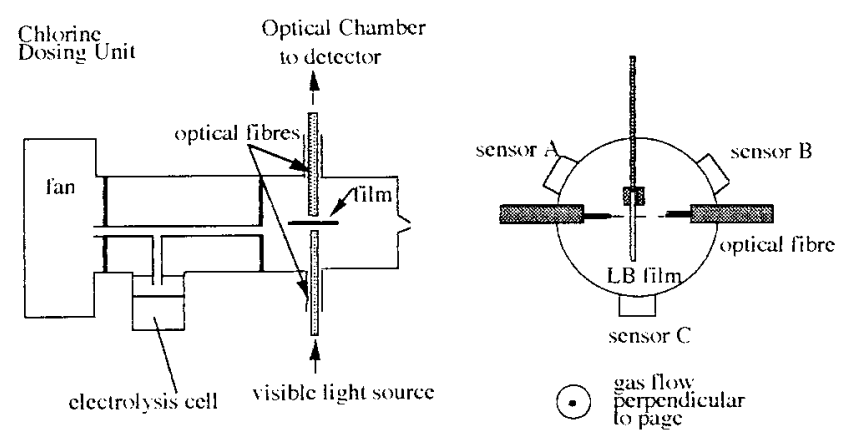

Fig. 2. Experimental arrangement for chlorine gas testing using in-situ UVvisible spectroscopy.

surface pressure $\left(8 \mathrm{mN} \mathrm{m}^{-1}\right)$ just below the first major phase transition of the $\pi-A$ curve. At this pressure the area per porphyrin molecule is $\sim 2.3 \mathrm{~nm}^{2}$, which corresponds closely to the cross-sectional area of the flat porphyrin disk. When organised in this way, the centres of the porphyrin rings are in the most accessible orientations with respect to incoming gas molecules.

\subsection{Concentration dependence of the initial response rate constant and the extent of recovery}

Fig. 4a depicts the variation in the initial rate constant as a function of chlorine concentration for monolayer films deposited at $8 \mathrm{mN} \mathrm{m}^{-1}$. The rate constant rises approximately linearly over the $0-2 \mathrm{ppm}$ range, above which it saturates. Although this plot represents only the initial response, Fig. $4 \mathrm{~b}$ shows that the percentage total change in Soret band absorbance ( $\{$ absorbance at saturation/initial absorbance $\} \times 100$ ) follows the same dependence on con-

(a)
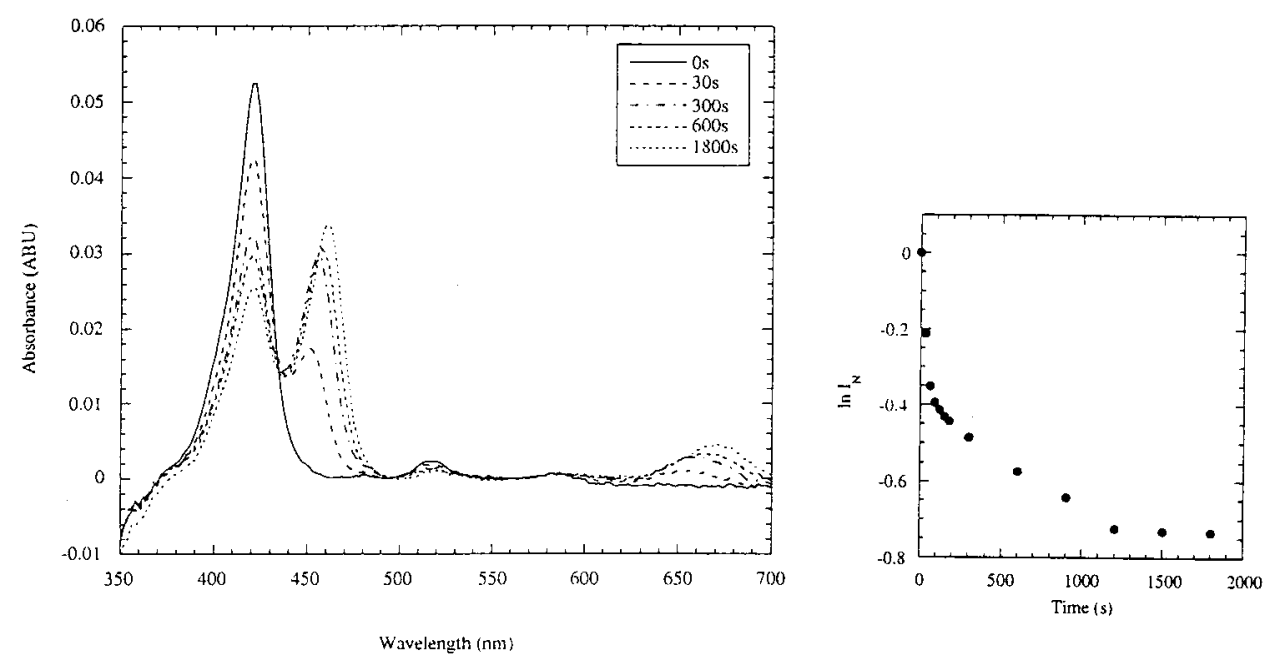

(b)
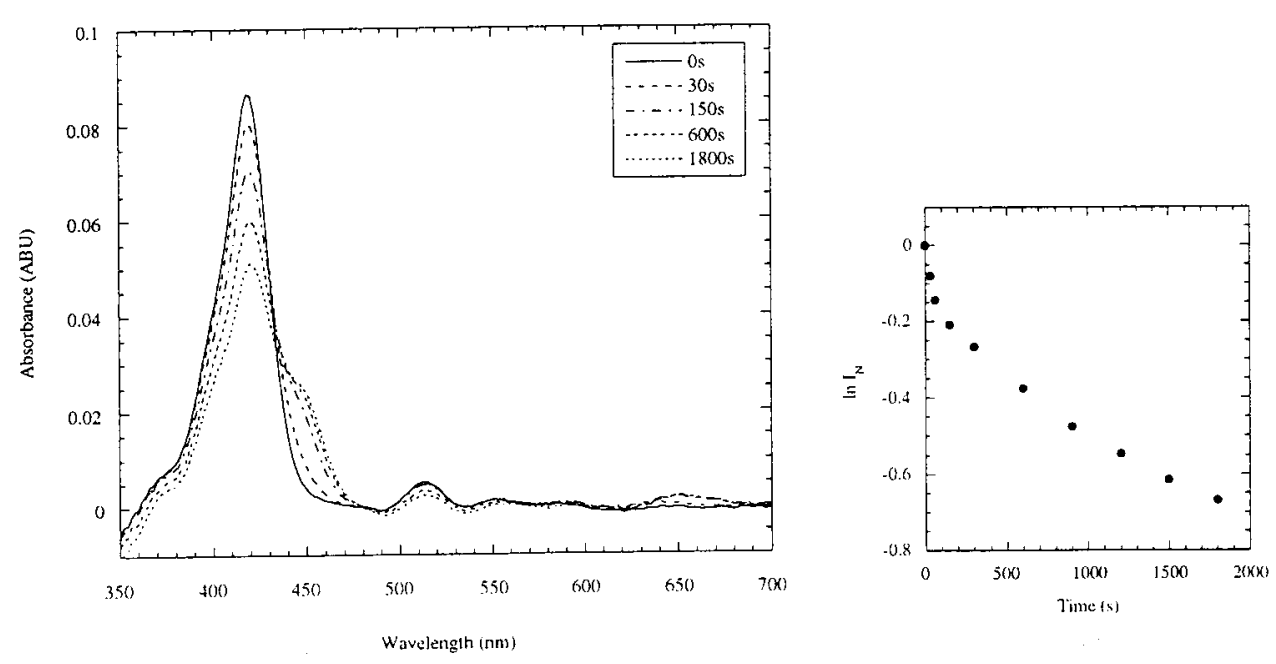

Fig. 3. Optical absorbance during exposure to $5 \mathrm{ppm}$ chlorine and corresponding logarithmic plot of normalised absorbance of Soret band versus time for a monolayer film of t-SAP-P deposited at (a) $8 \mathrm{mN} \mathrm{m}^{-1}$ and (b) $16 \mathrm{mN} \mathrm{m}^{-1}$. 
(a)

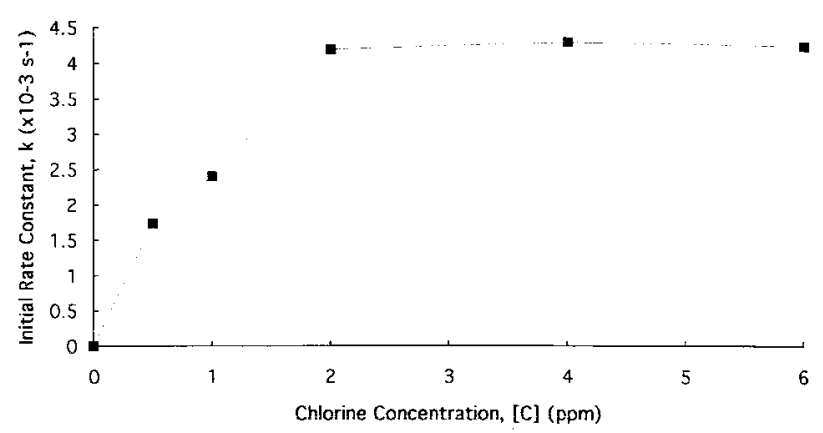

(b)

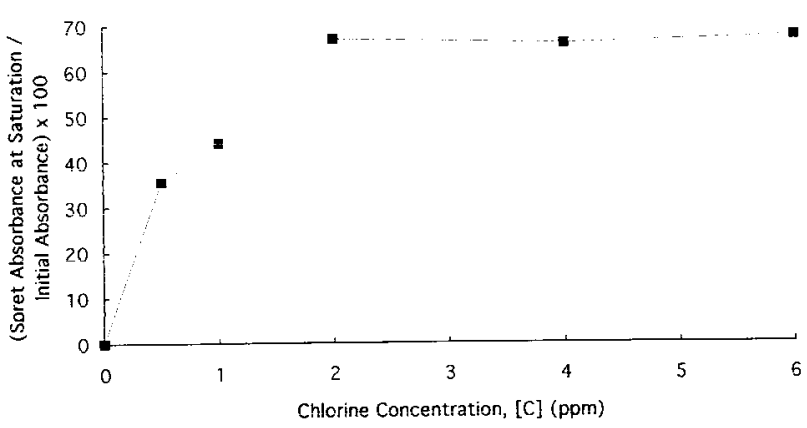

Fig. 4. Concentration dependence of (a) the initial rate constant, $k$, and (b) percent reduction in the Soret band intensity for a monolayer film of tSAP-P deposited at $8 \mathrm{mN} \mathrm{m}^{-1}$.

centration. This confirms that the initial rate constant is a good indicator of the total gas response for these films.

\subsection{Repeated dosing}

The effect of repeatedly dosing monolayer films deposited at $8 \mathrm{mN} \mathrm{m}^{-1}$ with $2 \mathrm{ppm}$ chlorine is shown in Fig. $5 \mathrm{a}$ for five consecutive doses each interspersed with a 20-h time period to facilitate full recovery (at room temperature). The $y$-axis $\left(I_{\mathrm{R}}\right)$ shows the relative change (compared to the Soret band) in the $455 \mathrm{~nm}$ peak versus time for both the response and recovery regimes. Encouragingly, reproducible behaviour is observed. However, the characteristics for three consecutive doses at a higher gas concentration show that the second and third doses yield a much less dramatic optical response than the initial exposure to chlorine. This suggests that exposure to high concentrations of chlorine leads to irreversible quenching of gas adsorption sites which are no longer able to contribute to the integrated optical response during further dosing.

\subsection{Accelerated recovery after heat treatment}

The use of elevated temperature in gas sensing applica- tions at pre-response, response and recovery stages has been reported previously [6-10]. Here, the effect of heating the porphyrin monolayers to $60^{\circ} \mathrm{C}$ during recovery will be discussed. Fig. 6a shows the spectral changes during a 900-s recovery period for (i) an unheated monolayer and (ii) a monolayer heated to $60^{\circ} \mathrm{C}$. The logarithmic plots in Fig. $6 \mathrm{~b}$ indicate that the return of the Soret band $\left(I_{\mathrm{N}}\right)$ towards its original intensity occurs at a much faster rate at elevated temperature compared to the recovery at ambient temperature and that recovery is complete after only $\sim 100 \mathrm{~s}$. Similarly, the disappearance of the $455 \mathrm{~nm}$ band $\left(I_{\mathrm{R}}\right)$ is much faster at $60^{\circ} \mathrm{C}$ and is complete at $\sim 200 \mathrm{~s}$. The acceleration in the recovery process from several hours to $100 \mathrm{~s}$ by moderate heating is staggering and is very encouraging for the future development of these materials as sensors.

\subsection{Effect of ageing}

The effect of long-term storage at room temperature in

(a)

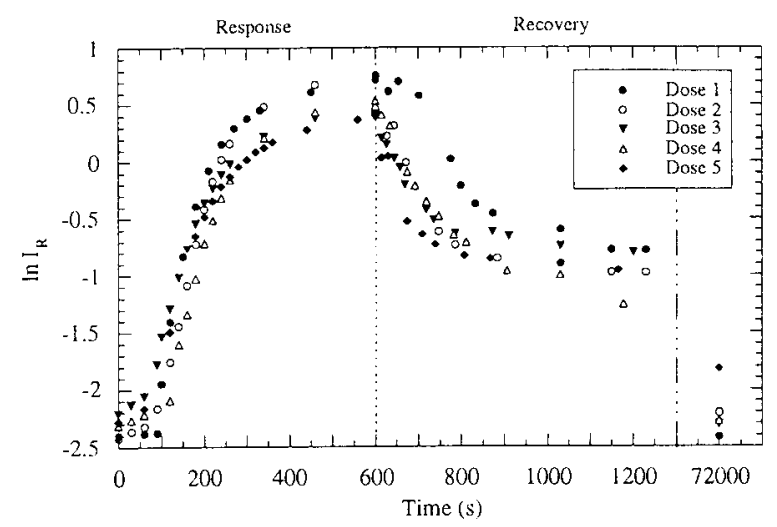

(b)

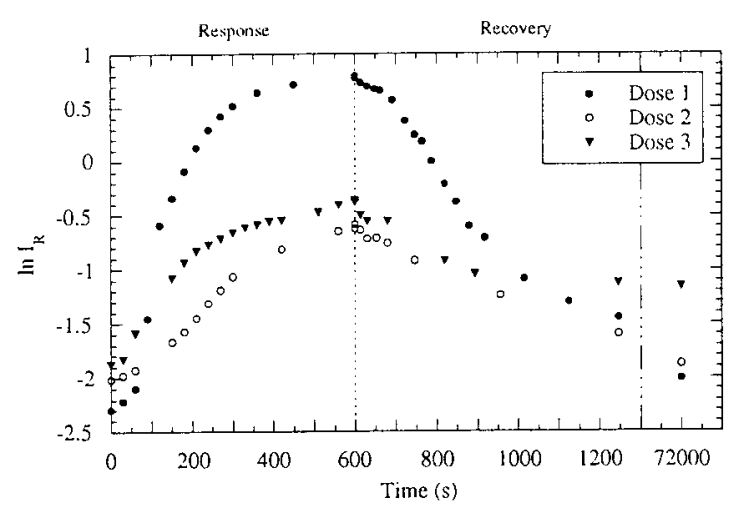

Fig. 5. Logarithmic plot of $455 \mathrm{~nm}$ band intensity (relative to Soret band intensity) versus time for (a) five consecutive doses of $2 \mathrm{ppm}$ chlorine and (b) three consecutive doses of $10 \mathrm{ppm}$ chlorine. 
(a)

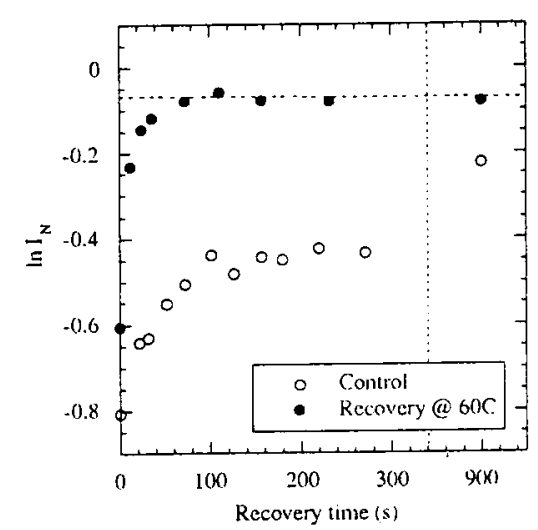

(b)

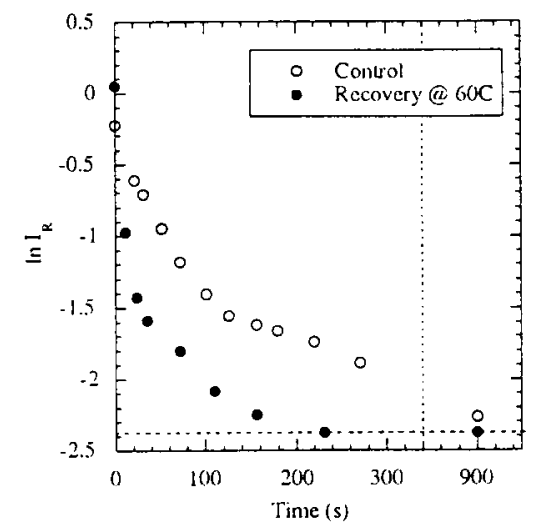

Fig. 6. Comparison of samples at room temperature and heat-treated during the recovery process. Logarithmic plot of (a) normalised Soret absorbance and (b) the $455 \mathrm{~nm}$ band absorbance (relative to the Soret band intensity) as a function of time.

(a)

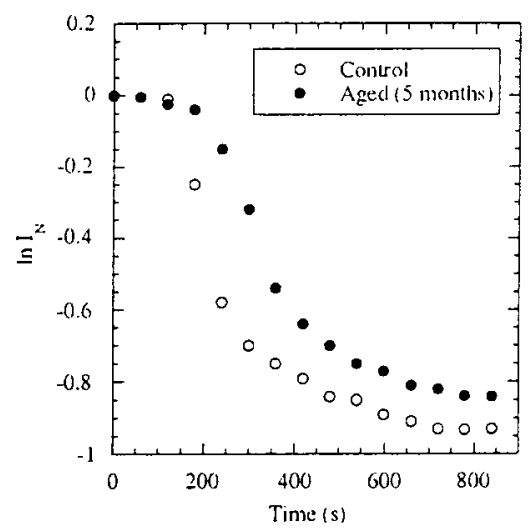

(b)

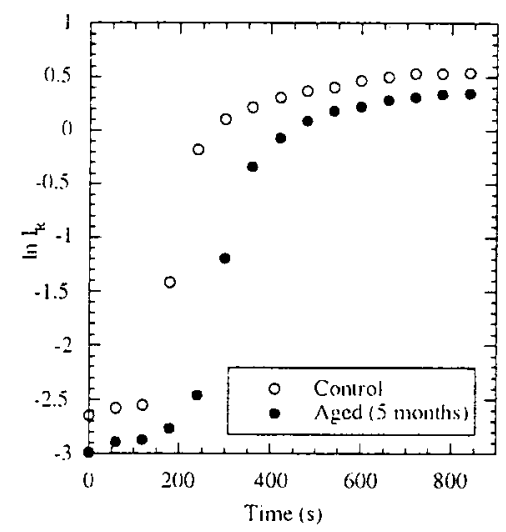

Fig. 7. Comparison of the chlorine gas response of fresh and aged (5 months) monolayers of t-SAP-P. Logarithmic plot of (a) normalised Soret absorbance and (b) $455 \mathrm{~nm}$ band absorbance (relative to Soret band intensity) as a function of time.

the dark on the gas response was investigated. Fig. 7 shows the intensity changes for (i) the Soret band and (ii) the 455 $\mathrm{nm}$ peak. The characteristics for the aged (5 months in the dark at atmospheric pressure and room temperature $\left.\left(\sim 20^{\circ} \mathrm{C}\right)\right)$ and freshly prepared samples are similar; the main difference is that the onset of the response takes longer to occur for the aged sample.

\section{Summary}

The effect of gaseous chlorine on the optical spectra of monolayers of metal-free meso-tetra(4-amino sulfonyl)phenylporphyrin has been described. Upon exposure to chlorine vapour in the concentration range $0.1-10 \mathrm{ppm}$, the Soret and Q-bands decrease in intensity and new bands at $\sim 455$ $\mathrm{nm}$ and $\sim 660 \mathrm{~nm}$ appear. The rate of response and the total extent of the optical response rise linearly over the concen- tration range $0-2$ ppm before saturation. Repeated dosing experiments have shown that reversible and reproducible sensing action occurs provided that a threshold concentration is not exceeded. Moderate heat treatment $\left(60^{\circ} \mathrm{C}\right.$ for 10 $\mathrm{min}$ ) brings about the recovery of the original optical spectrum in only $\sim 100 \mathrm{~s}$ compared with several hours at room temperature. Ageing experiments have shown that the basic response of the monolayer is not effected over a time period of at least 5 months.

\section{References}

[1] S. Baker, G.G. Roberts, M.C. Petty, Proc. IEE Part I: Solid State Electron. Devices 130 (1983) 260.

[2] A.K. Ray, M.J. Cook, S.C. Thorpe, S. Mukhopadyay, Phys. Stat. Sol. (a) 140 (1993) K85.

[3] V.C. Smith, S.V. Batty, T. Richardson, K.A. Foster, D. Bonnick, 
R.A.W. Johnstone, A. Sobral, A.M.d'A. Rocha-Gonsalves, Thin Solid Films 284 (1996) 911.

[4] M.B. Grieve, A.J. Hudson, T. Richardson, R.A.W. Johnstone, A.J.F.N. Sobral, A.M.d'A. Rocha-Gonsalves, Thin Solid Films 243 (1994) 581.

[5] M.B. Grieve, Ph.D. Thesis, University of Sheffield, UK, 1995.

[6] P.B.M. Archer, A.V. Chadwick, J.J. Miasik, M. Tamizi, J.D. Wright, Sens. Actuators 16 (1989) 379.
[7] Y. Sadaoka, M. Matsuguchi, Y. Sakai, Y. Mori, J. Mater. Sci. 27 (1992) 5215.

[8] M.J. Cook, Int. J. Electron. 76 (1994) 727.

[9] T.A. Jones, B. Bott, S.C. Thorpe, Sens. Actuators 17 (1989) 467.

[10] Y. Sadaoka, T.A. Jones, W. Gopel, Sens. Actuators B 1 (1990) 148. 